\title{
Enhanced Virulence of Candida albicans by Staphylococcus aureus: Evidence in Clinical Bloodstream Infections and Infected Zebrafish Embryos
}

\author{
Yen-Mu Wu ${ }^{1,2,+}$, Po-Yen Huang ${ }^{1,+}$, Yi-Chuan Cheng ${ }^{3,4}$, Chih-Hua Lee ${ }^{5}$, Meng-Chieh Hsu ${ }^{6}$, Jang-Jih Lu ${ }^{5,7,8, *}$ \\ and Shao-Hung Wang ${ }^{6, *}$ id
}

1 Department of Internal Medicine, Division of Infectious Diseases, Chang Gung Memorial Hospital at Linkou Medical Center, Taoyuan City 333, Taiwan; yenmuwu@gmail.com (Y.-M.W.); pyhuang@gmail.com (P.-Y.H.)

2 Graduate Institute of Clinical Medical Sciences, College of Medicine, Chang Gung University, Taoyuan City 333, Taiwan

3 Graduate Institute of Biomedical Sciences, College of Medicine, Chang Gung University, Taoyuan City 333, Taiwan; yccheng@mail.cgu.edu.tw

4 Neuroscience Research Center, Chang Gung Memorial Hospital at Linkou Medical Center, Taoyuan City 333, Taiwan

5 Department of Laboratory Medicine, Chang-Gung Memorial Hospital at Linkou Medical Center, Taoyuan City 333, Taiwan; josh60708@cgmh.org.tw

6 Department of Microbiology, Immunology and Biopharmaceuticals, National Chiayi University, Chiayi City 600, Taiwan; a23235630@gmail.com

7 Department of Medical Biotechnology and Laboratory Science, Chang Gung University, Taoyuan City 333, Taiwan

check for updates

Citation: Wu, Y.-M.; Huang, P.-Y.; Cheng, Y.-C.; Lee, C.-H.; Hsu, M.-C.; Lu, J.-J.; Wang, S.-H. Enhanced Virulence of Candida albicans by Staphylococcus aureus: Evidence in Clinical Bloodstream Infections and Infected Zebrafish Embryos. J. Fungi 2021, 7, 1099. https://doi.org/ 10.3390/jof7121099

Academic Editor: Mathieu Nacher

Received: 19 November 2021 Accepted: 16 December 2021 Published: 20 December 2021

Publisher's Note: MDPI stays neutral with regard to jurisdictional claims in published maps and institutional affiliations.

Copyright: (c) 2021 by the authors. Licensee MDPI, Basel, Switzerland. This article is an open access article distributed under the terms and conditions of the Creative Commons Attribution (CC BY) license (https:/ / creativecommons.org/licenses/by/ $4.0 /)$.
8 Department of Medicine, College of Medicine, Chang Gung University, Taoyuan City 333, Taiwan

* Correspondence: janglu45@gmail.com (J.-J.L.); shwang@mail.ncyu.edu.tw (S.-H.W.); Tel.: +886-3-3281200 (ext. 2554) (J.-J.L.); +886-5-2717225 (S.-H.W.)

+ Authors with equal contribution.

\begin{abstract}
Coinfection with Candida and Staphylococcus results in higher mortality in animal studies. However, the pathogenesis and interplay between C. albicans and S. aureus in bloodstream infections (BSIs) is unclear. This study determines the clinical features and outcomes of mixed C. albicans/S. aureus (CA/SA) BSIs and biofilm formation on pathogenesis during coinfection. Demographics and outcomes for mixed BSIs and monomicrobial candidemia were compared. Compared to 115 monomicrobial C. albicans BSIs, 22 patients with mixed CA/SA BSIs exhibited a significantly higher mortality rate and shorter survival time. In vitro and in vivo biofilm analysis showed that $C$. albicans accounted for the main biofilm architecture, and S. aureus increased its amount. Antibiotic tolerance in S. aureus, which adhered to Candida hyphae observed by scanning electron microscope, was demonstrated by the presence of wild-type C. albicans co-biofilm. Upregulation in exotoxin genes of $S$. aureus was evidenced by quantitative RT-PCR when a co-biofilm was formed with C. albicans. Mixed CA/SA BSIs result in a higher mortality rate in patients and in vivo surrogate models experiments. This study demonstrates that the virulence enhancement of C. albicans and S. aureus during co-biofilm formation contributes to the high mortality rate.
\end{abstract}

Keywords: mixed BSI; virulence; co-biofilm; Candida albicans; Staphylococcus aureus; hyphal morphogenesis; zebrafish embryo infection

\section{Introduction}

The global SENTRY Antimicrobial Surveillance Program from 2012-2017 demonstrated that Staphylococcus aureus, which was the most prevalent bacterial pathogen, caused $22.5 \%$ of bloodstream infections (BSIs), and Candida species caused 3.1\% [1], with increasing prevalence during the last decade [2]. Mixed BSIs with bacteria and Candida results in 
a more severe prognosis and are associated with higher morbidity and mortality than monomicrobial or polybacterial BSIs [3-5].

S. aureus and C. albicans are both important lethal pathogens in nosocomial BSIs, and there is an increase in the incidence of mixed BSI, so the risk factors and prognosis of mixed C. albicans/S. aureus (CA/SA) BSIs in the population and the interplay between C. albicans and $S$. aureus in host pathogenesis are worthy of investigation.

Coinfection models involving Candida and Staphylococcus have been used in several animal studies [6-12]. These studies all show that mixed-infection with Candida and Staphylococcus increases mortality of the infected hosts, and the interplay between the bacterium and the fungus significantly increases the bacterial burden or virulence on hosts. It has been demonstrated that a biofilm of Candida protects bacteria from host defensiveness, and each microbial secretory factor affects their virulence [13,14].

Embryos of zebrafish are commonly used as a surrogate infection host and are used as a model to determine the biofilm activity of $C$. albicans in vivo. This model allows antifungal screening and genetic studies with the advantages of conserved immunity, prolific fecundity, and cost-effectiveness [15-18]. In order to determine the interplay between $C$. albicans and S. aureus in infected hosts without immune interference, a zebrafish embryo infection assay was used for this study.

\section{Materials and Methods}

\subsection{Study Design and Definition}

This retrospective study was conducted from 2003 to 2015 at Chang Gung Memorial Hospital at Linkou (CGMHL) and was approved by the institutional review board of the hospital (201701182B0 and 202101345B0). Adult hospitalized patients ( $\geq 18$ years old) were recruited. Mixed CA/SA BSI cases were defined by a positive blood culture for $C$. albicans with $S$. aureus growing concomitantly from the same set. Data from adult patients who had monomicrobial C. albicans BSIs in 2010 were used for comparison.

\subsection{Clinical Characteristics and Outcomes}

Data for demographics, comorbidities, risk factors, and clinical outcomes were recorded. Comorbidities included heart failure, chronic lung diseases, diabetes mellitus, hepatic dysfunction (a serum total bilirubin level $\geq 2.0 \mathrm{mg} / \mathrm{dL}$ or liver cirrhosis), renal insufficiency (a serum creatinine level $\geq 2.0 \mathrm{mg} / \mathrm{dL}$ or a requirement for hemodialysis), hematological malignancies, and solid tumors. The risk factors collected within 30 days before the BSIs included abdominal surgery, central venous catheterization, parenteral nutrition, immunosuppressant administration (prednisolone $\geq 20 \mathrm{mg} /$ day for more than 3 weeks, chemotherapeutic and other immunosuppressive drugs), and neutropenia (an absolute neutrophil count $<500$ cells $/ \mathrm{mL}$ ). Severity of illness was assessed at the onset of BSIs using a sequential organ failure assessment (SOFA) score [19]. Clinical outcomes were assessed in terms of mortality, median survival days after BSIs, and length of stay in hospital. The clinical characteristics and outcomes for $C$. albicans candidemia were described in a previous study by the authors [20].

\subsection{Microbes and Fish Strains}

To evaluate the virulence enhancement of $C$. albicans biofilm by $S$. aureus, a virulent laboratory strain SC5314 and its biofilm-defective mutant HLC54 were chosen as well as a hemolysin-producing S. aureus ATCC 29213, which is the most commonly used strain in the clinical laboratory. C. albicans HLC54 (cph1/cph1 efg1/efg1) is a hyphae-defective mutant derived from virulent parental SC5314 [21]. A clinical isolate P004 with low biofilm was also used as the fungal pathogen [17]. All yeasts are caspofungin susceptible. A methicillinsusceptible S. aureus ATCC 29213 was used as the bacterial pathogen, which is vancomycin susceptible [22]. Wild-type zebrafish (Danio rerio) AB line (Zebrafish International Resource Center, Eugene, OR, USA), aged approximately $8-15$ months, were maintained at $28^{\circ} \mathrm{C}$ in a 10-h dark 14-h light cycle to collect fertilized eggs, which served as surrogate hosts. 
All experiments were performed in accordance with standard guidelines for zebrafish studies [23].

\subsection{In Vitro Biofilm Analysis}

3-Morpholinopropane sulfonic acid (MOPS)-buffered RPMI-1640 (pH7.2) containing $10 \%$ fetal calf serum and $1.25 \% \mathrm{~N}$-acetyl-D-glucosamine was used as a biofilm formation medium. For the metabolic reducing dye assay, overnight-cultivated microbes were refreshed for $4 \mathrm{~h}, 10^{5} \mathrm{cfu}$ of the cultures were seeded into microplates, and then, biofilm that was cultured at $37^{\circ} \mathrm{C}$ for 4-h or 24-h was measured using a CCK-8 kit (cat. 96992, Sigma-Aldrich, St. Louis, MO, USA). For the other biofilm quantification method using safranin, sterile 13-mm hydrophilic filters (cat. AAWP01300, Millipore, Bedford, MA, USA) were used as an adherent surface, and biofilm-stained Gram's safranin was dissolved using $30 \%$ acetic acid and measured using $\mathrm{OD}_{530}$.

\subsection{Zebrafish Embryo Infection Assay}

The infection assay follows the method previously described [17]. Embryos were co-incubated with $5 \times 10^{5}$ yeast $/ \mathrm{mL} \mathrm{C}$. albicans and $/$ or $2 \times 10^{7} \mathrm{cfu} / \mathrm{mL} \mathrm{S}$. aureus in RPMI-1640 medium (Thermo Fisher Scientific, Waltham, MA, USA) at $120 \mathrm{rpm} 30^{\circ} \mathrm{C}$ for $4 \mathrm{~h}$. After 4-h co-incubation, the infected embryos were washed thrice with egg water $(0.03 \%$ sea salt) to remove non-adherent microbes and then transferred into fresh egg water supplemented with $0.5 \%$ YPD broth and were grown at $30^{\circ} \mathrm{C}$ for $24 \mathrm{~h}$. The survival rate was calculated as the percentage of the death by monitoring the heartbeat of embryos. All experiments were approved by the Biosafety Committee (No. 00417-2020092830581) and IACUC (CGU109-109) of the hospital and were conducted in a BSL-2 laboratory.

\subsection{Microbial Enumeration}

After 4-h adhesion, the microbial infected chorions were incubated for $20 \mathrm{~h}$ to allow biofilm maturation and were treated with antimicrobials $(80 \mu \mathrm{g} / \mathrm{mL}$ vancomycin or $0.5 \mu \mathrm{g} / \mathrm{mL}$ caspofungin). The chorions were washed and transferred to microtubes and ground using a pestle. The resulting mixtures were colony counted for microbes on suitable agar plates with vancomycin $(4 \mu \mathrm{g} / \mathrm{mL})$ or caspofungin $(4 \mu \mathrm{g} / \mathrm{mL})$.

\subsection{Scanning Electron Microscope (SEM)}

Samples were washed and harvested after 24-h incubation and fixed in a mixture of $3 \%$ gutaraldehyde and $2 \%$ paraformaldehyde that was buffered to $\mathrm{pH} 7.4$ using $0.1 \mathrm{M}$ cacodylate buffer. Samples were washed with cacodylate buffer and treated with $1 \%$ osmium tetroxide. The cell pellets were washed thoroughly with $0.1 \mathrm{M}$ cacodylate buffer and dehydrated gradually with ethanol until only $100 \%$ ethanol remained. After criticalpoint drying and mounting on an SEM stub, the samples were sputter-coated with a thin layer of gold, and images were recorded at an appropriate accelerating voltage.

\subsection{Gene Expression Assays}

The biofilm mass was dissolved and ground in REzol C\&T reagent (Protech Technology, Taipei, Taiwan) and kept frozen before RNA extraction. To determine the expression of $S$. aureus toxin genes in the biofilm, total RNA extracted with MagNA Pure Compact RNA Isolation Kit (Roche Applied Science, Indianapolis, IN, USA) was two-step reversetranscribed (High Capacity cDNA Reverse Transcription Kit, Applied Biosystems, Foster City, CA, USA) to cDNA and analyzed by quantitative PCR run in ABI 7900 HT Real-Time PCR System: $10 \mathrm{~min}$ at $95^{\circ} \mathrm{C}$ and 40 cycles of $15 \mathrm{sec}$ at $95^{\circ} \mathrm{C}, 1 \mathrm{~min}$ at $60^{\circ} \mathrm{C}$ using $2 \times$ Gene Expression Master Mix (Applied Biosystems, Foster City, CA, USA). The primers (Mission Biotech, Taipei, Taiwan) and TaqMan probes (Thermo Fisher Scientific, Waltham, MA, USA) corresponding to $S$. aureus housekeeping gene $r r s A$ and hemolysin genes $h l a$ and $h l g B$ are listed in Table S1. The change in the expression was calculated using the comparative CT method, with $\operatorname{rrs} A$ as an endogenous control. 


\subsection{Statistical Analysis}

Statistical analysis used SPSS 22.0 software (IBM, Armonk, NY, USA). Continuous variables are presented as mean \pm standard deviation (SD) if the data are normally distributed and as a median and interquartile range (IQR) if the data are non-normally distributed. Categorical variables were compared using Pearson's chi-square test or Fisher's exact test and continuous variables were compared using a Student's t-test or a Mann-Whitney U test. Variables with a two-tailed $p$-value $<0.01$ were included in a binary logistic regression model for a multivariate analysis. All tests were two-tailed, and a $p$-value of $<0.05$ represents statistical significance.

\section{Results}

\subsection{Clinical Characterization of Mixed C. albicans/S. aureus BSIs}

A total of 22 patients with mixed CA/SA BSIs from 2003-2015 and 115 monomicrobial C. albicans BSI patients in 2010 at CGMHL were included in this study. The demographics, clinical characteristics, risk factors, and outcomes were compared. More patients in mixed CA/SA BSI group exhibited renal insufficiency (59.1\% vs. $27.0 \%, p=0.003)$. Unlike $C$. albicans candidemia, mixed CA/SA BSI cases were associated with a higher SOFA score (10.6 vs. $7.0, p=0.003)$, higher 14 -/30-day mortality rate (14-day: $77.3 \%$ vs. $40.0 \%, p=0.002$; 30 -day: $81.8 \%$ vs. $53.9 \%, p=0.018$ ), and shorter median survival days (4.5 vs. 25.0, $p=0.001$ ) (Table 1 ). Within 22 mixed CA/SA BSIs, five patients died within $24 \mathrm{~h}$ after blood cultures were obtained. Of the remaining 17 patients, intravascular catheters were removed in $13(76.5 \%)$, and follow-up blood cultures were performed in five $(29.4 \%)$, yielding no growth of C. albicans or S. aureus.

Patients who did not survive more than 14 days were correlated with liver dysfunction $(39.7 \%$ vs. $13.5 \%, p<0.001)$, renal insufficiency $(52.4 \%$ vs. $14.9 \%, p<0.001)$, higher SOFA score (11 vs. $4, p<0.001)$, and more mixed CA/SA BSIs $(27.0 \%$ vs. $6.8 \%, p=0.001)$ (Table 2$)$. Multivariate analysis showed that 14-day mortality was positively associated with a high SOFA score (OR 1.13, 95\% CI 1.03-1.24) and mixed CA/SA BSIs (OR 3.47, 95\% CI 1.05-11.50) (Table 3).

Table 1. Comparison of clinical features and outcomes for patients in mixed CA/SA BSI and C. albicans candidemia groups.

\begin{tabular}{lccc}
\hline \multicolumn{1}{c}{ Variables } & $\begin{array}{c}\text { Mixed CA/SA } \\
\text { BSI }\end{array}$ & $\begin{array}{c}\text { C. albicans } \\
\text { Candidemia }\end{array}$ & Univariate \\
\hline & $\boldsymbol{N}=\mathbf{2 2}$ & $\boldsymbol{N}=\mathbf{1 1 5}$ & $p$-Value \\
Age (SD), years & $73.1(15.0)$ & $66.5(14.5)$ & 0.053 \\
Sex, male & $7(31.8)$ & $64(55.7)$ & 0.061 \\
\hline Comorbidities & & & \\
\hline Cardiovascular disease & $4(18.2)$ & $16(13.9)$ & 0.530 \\
Chronic lung disease & $7(31.8)$ & $17(14.8)$ & 0.068 \\
Diabetes mellitus & $9(40.9)$ & $37(32.2)$ & 0.427 \\
Liver dysfunction & $6(27.3)$ & $29(25.2)$ & 0.796 \\
Renal insufficiency & $13(59.1)$ & $31(27.0)$ & 0.003 \\
Hematological malignancy & $1(4.5)$ & $6(5.2)$ & 1.000 \\
Solid tumor & $3(13.6)$ & $64(55.7)$ & 0.000 \\
\hline
\end{tabular}


Table 1. Cont.

\begin{tabular}{|c|c|c|c|}
\hline Variables & $\begin{array}{c}\text { Mixed CA/SA } \\
\text { BSI }\end{array}$ & $\begin{array}{l}\text { C. albicans } \\
\text { Candidemia }\end{array}$ & Univariate \\
\hline \multicolumn{4}{|l|}{ Risk factors ${ }^{1}$} \\
\hline Abdominal surgery & $3(13.6)$ & $19(16.5)$ & 1.000 \\
\hline Intravascular catheter & $20(90.9)$ & $107(93.0)$ & 0.663 \\
\hline Parenteral nutrition & $4(18.2)$ & $60(52.2)$ & 0.004 \\
\hline Immunosuppressants & $2(9.1)$ & $38(33.0)$ & 0.023 \\
\hline Neutropenia $^{2}$ & $1(4.5)$ & $6(5.2)$ & 1.000 \\
\hline \multicolumn{4}{|l|}{ Clinical condition } \\
\hline SOFA score (SD) & $10.6(6.7)$ & $7.0(5.7)$ & 0.003 \\
\hline LOS before BSIs (SD), days & $48.5(50.7)$ & $34.2(29.6)$ & 0.309 \\
\hline \multicolumn{4}{|l|}{ Clinical outcomes } \\
\hline 14-day mortality & $17(77.3)$ & $46(40.0)$ & 0.002 \\
\hline 30-day mortality & $18(81.8)$ & $62(53.9)$ & 0.018 \\
\hline Median survival days (IQR) & $4.5(1.75-11.00)$ & $25.0(16.8-33.2)$ & 0.001 \\
\hline LOS (SD), days & $64.1(51.5)$ & $58.8(42.4)$ & 0.606 \\
\hline \multicolumn{4}{|c|}{$\begin{array}{l}\text { Note: Categorical data are presented as no. (\%) of subject. Mean (standard deviation (SD)) and media } \\
\text { (interquartile range (IQR)) are, respectively, used to describe normally and non-normally distributed dat } \\
\text { CA, Candida albicans; SA, Staphylococcus aureus; BSI, bloodstream infection; LOS, length of stay; SOFA, sequenti } \\
\text { organ failure assessment. }{ }^{1} \text { Risk factors were evaluated within } 30 \text { days before BSI occurrence. }{ }^{2} \text { An absolut } \\
\text { neutrophil count }<500 \text { cells } / \mathrm{mL} \text {. }\end{array}$} \\
\hline \multirow[t]{2}{*}{ Variables } & 14-D Mortality & 14-D Non-Mortality & Univariate \\
\hline & $N=63$ & $N=74$ & $p$-Value \\
\hline \multicolumn{4}{|l|}{ Comorbidities } \\
\hline Cardiovascular disease & $10(15.9)$ & $10(13.5)$ & 0.697 \\
\hline Chronic lung disease & $10(15.9)$ & $14(18.9)$ & 0.640 \\
\hline Diabetes mellitus & $24(38.1)$ & $22(29.7)$ & 0.301 \\
\hline Liver dysfunction & $25(39.7)$ & $10(13.5)$ & 0.000 \\
\hline Renal insufficiency & $33(52.4)$ & $11(14.9)$ & 0.000 \\
\hline Hematological malignancy & $4(6.3)$ & $3(4.1)$ & 0.703 \\
\hline Solid tumor & $25(39.7)$ & $42(56.8)$ & 0.046 \\
\hline \multicolumn{4}{|l|}{ Clinical scenarios } \\
\hline Abdominal surgery & $11(17.5)$ & $11(14.9)$ & 0.680 \\
\hline Intravascular catheter & $60(95.2)$ & $67(90.5)$ & 0.342 \\
\hline Parenteral nutrition & $30(47.6)$ & $34(45.9)$ & 0.845 \\
\hline Immunosuppressants & $18(28.6)$ & $22(29.7)$ & 0.882 \\
\hline Neutropenia & $2(3.2)$ & $5(6.8)$ & 0.452 \\
\hline SOFA score (IQR) & $11(4-15)$ & $4(1-7)$ & 0.000 \\
\hline Mixed CA/SA BSI & $17(27.0)$ & $5(6.8)$ & 0.001 \\
\hline LOS before BSI (SD), days & $43.9(40.4)$ & $34.2(29.7)$ & 0.110 \\
\hline
\end{tabular}

Table 3. Multivariate analysis of high variance factors that are associated with 14-day mortality for patients with BSIs.

\begin{tabular}{lcccc}
\hline \multicolumn{1}{c}{ Variables } & 14-D Mortality & $\begin{array}{c}\text { 14-D } \\
\text { Non-Mortality }\end{array}$ & \multicolumn{2}{c}{ Multivariate } \\
& $N=63$ & $N=74$ & OR (95\% CI) & $p$-Value \\
Liver dysfunction & $25(39.7)$ & $10(13.5)$ & $1.96(0.70-5.48)$ & 0.199 \\
Renal insufficiency & $33(52.4)$ & $11(14.9)$ & $2.18(0.80-5.91)$ & 0.126 \\
SOFA score (IQR) & $11(4-15)$ & $4(1-7)$ & $1.13(1.03-1.24)$ & 0.009 \\
Mixed CA/SA BSI & $17(27.0)$ & $5(6.8)$ & $3.47(1.05-11.50)$ & 0.041 \\
\hline
\end{tabular}




\subsection{Co-Biofilm of C. albicans and S. aureus In Vitro}

The in vitro biofilm activity assay was performed using a metabolic reducing dye and Gram's safranin staining. The results for the two biofilm assays showed that there was significantly higher activity in the mixed-infection group than in the monomicrobial groups (Figure 1). The difference of biofilm in 4-h adhesion between the mixed-infection group and others was more significant than that for the 24-h maturation, and the results showed that C. albicans accounted for most biofilm structure in terms of both the activity and the amount of biofilm. $\beta$-glucan in culture supernatants of $C$. albicans has been shown to potentiate drug resistance of $S$. aureus [24]. Biofilm enhancement in microbial supernatants was also studied, and a significant increase in S. aureus biofilm with C. albicans supernatants was observed in terms of the activity and the amount (Figure S1).

(A)

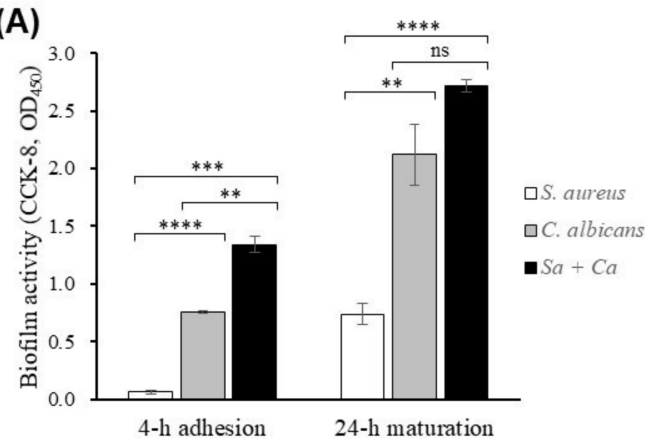

(B)

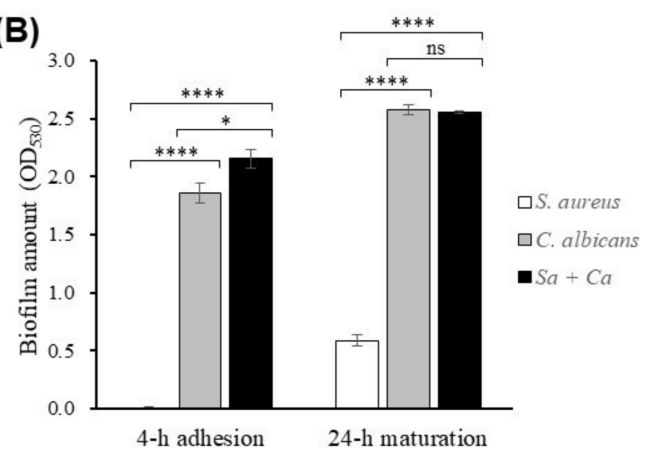

Figure 1. Analysis of in vitro microbial biofilm with C. albicans and S. aureus. Using microplates or hydrophilic membrane filters as an adherent surface, $10^{5} \mathrm{cfu}$ of $C$. albicans and/or S. aureus strains were seeded for 4-h adhesion and 24-h maturation of biofilms at $37{ }^{\circ} \mathrm{C}$ and analyzed using a CCK-8 kit (A) or Gram's safranin dye staining (B). Note: ${ }^{*} p<0.05,{ }^{* *} p<0.01,{ }^{* * *} p<0.001,{ }^{* * * *} p<0.0001$, ns $=$ not significant.

\subsection{Enhancement of Virulence of C. albicans Biofilm by S. aureus on Zebrafish Embryos}

Within 4-h incubation, the microbial loads for S. aureus and C. albicans SC5314 grown on the surface of egg chorion were, respectively, calculated to be $1.5 \times 10^{6} \mathrm{cfu}$ and $3.5 \times 10^{6} \mathrm{cfu}$ per egg (data not shown). After 24-h development, no matter whether S. aureus was present, heavy and thick biofilms were observed on chorions of the SC5314 group and the lowbiofilm P004 group (Figure 2K,L,O,P), but there was no obvious biofilm observed in neither S. aureus nor hyphae-defective HLC54-infected groups (Figure 2J,M,N).

Co-cultivation of C. albicans significantly increased the bacterial load in the co-biofilm on eggs from $2.3 \times 10^{7}$ to $6.9 \times 10^{7}$ in the SC5314 group and $2.6 \times 10^{8}$ in the HLC54 group (Table 4). Significantly fewer embryos in the co-infected groups survived and this reduction was much greater for wild-type SC5314: from 35\% to 7\% (Figure 2L,P). 


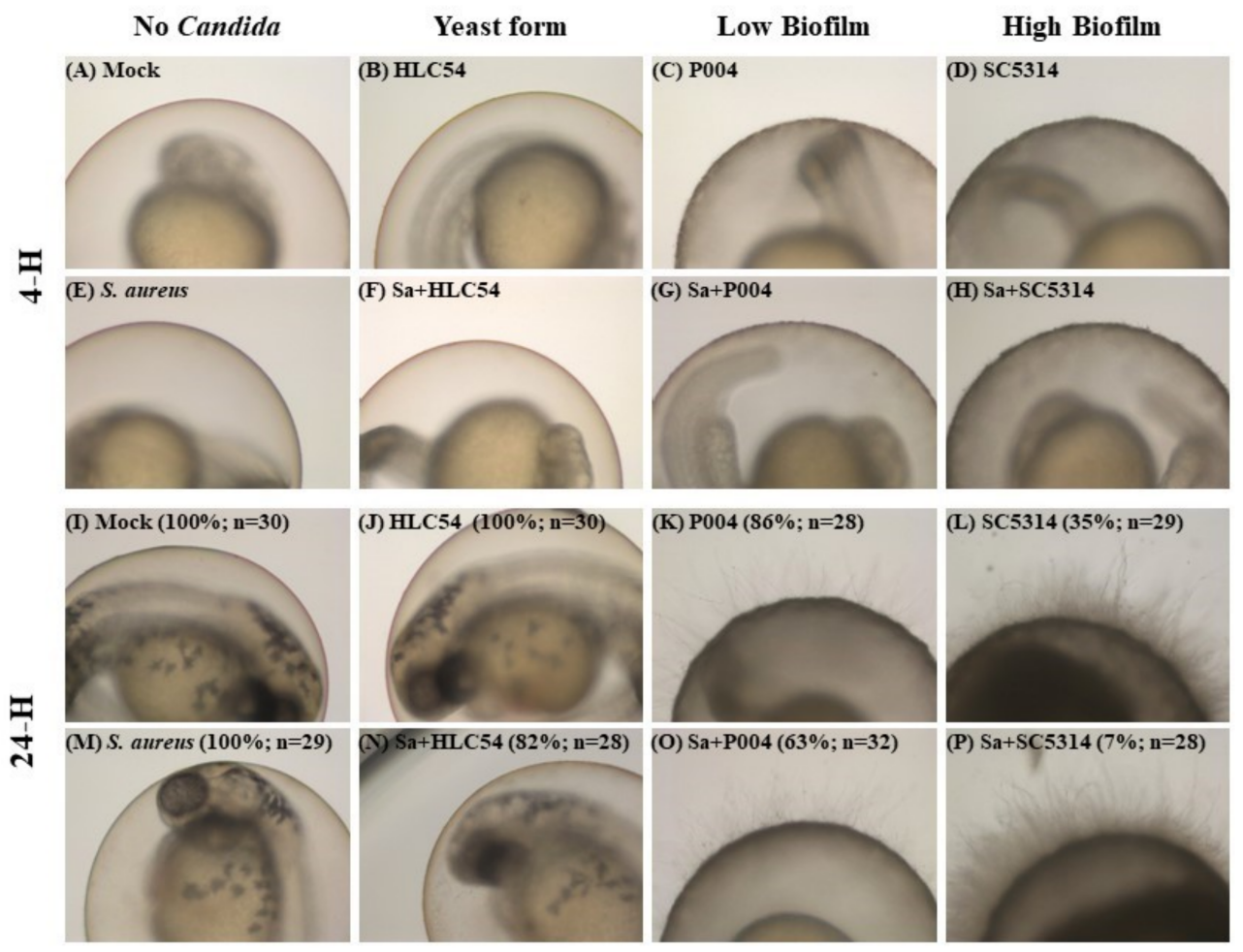

Figure 2. Analysis of in vivo microbial biofilms on zebrafish chorions with C. albicans and S. aureus. After 4-h adhesion, bound microorganisms, including S. aureus ATCC 29213, C. albicans hyphaedefective mutant HLC54, low biofilm clinical strain P004, and wild-type SC5314 strains, were cultivated at $30{ }^{\circ} \mathrm{C}$ for $24 \mathrm{~h}$ to form mature biofilms (A-P). Photographs were taken during the 4-h adhesion and 24-h maturation periods. The survival rate for infected embryos was measured and is shown in parentheses with the number $(n)$ of eggs that was used for each experiment. Around 30 embryos were used for each test. The heart-rate of fish embryos was measured to determine the survival rate.

Table 4. Protective strength of S. aureus from antibiotic assault due to C. albicans in the co-biofilm.

\begin{tabular}{|c|c|c|}
\hline Treat & Infection Microorganisms & S. aureus Load (cfu/egg) \\
\hline \multirow{6}{*}{ No Vancomycin } & Mock infection & 0 \\
\hline & C. albicans HLC54 & 0 \\
\hline & C. albicans SC5314 & 0 \\
\hline & S. aureus & $2.3 \times 10^{7}$ \\
\hline & S. aureus + HLC54 & $2.6 \times 10^{8}$ \\
\hline & S. aureus + SC5314 & $6.9 \times 10^{7}$ \\
\hline \multirow{3}{*}{ Vancomycin } & S. aureus & 0 \\
\hline & S. aureus + HLC54 & 0 \\
\hline & S. aureus + SC5314 & $3.0 \times 10^{7}$ \\
\hline
\end{tabular}

\subsection{Protection of S. aureus against Antibiotics in a Co-Biofilm with Hyphal C. albicans}

The $S$. aureus loads after vancomycin treatment were significantly increased in cobiofilm with wild-type SC5314, while the protection declined when formed with a hyphaedefective HLC54 (Table 4). However, no obvious protective effect was observed in the co-biofilms for C. albicans against caspofungin, either SC5314 or HLC54 (data not shown). The scanning electron microscope (SEM) results showed that $S$. aureus almost adhered 
to C. albicans hyphae (Figure 3), but yeast cells did not express molecules for intimate interaction with S. aureus to shield from drug damage. There was no obvious change in the biofilm morphology for co-infected embryos that were treated with vancomycin, which eliminated non-hyphal protected S. aureus (Figure 4).
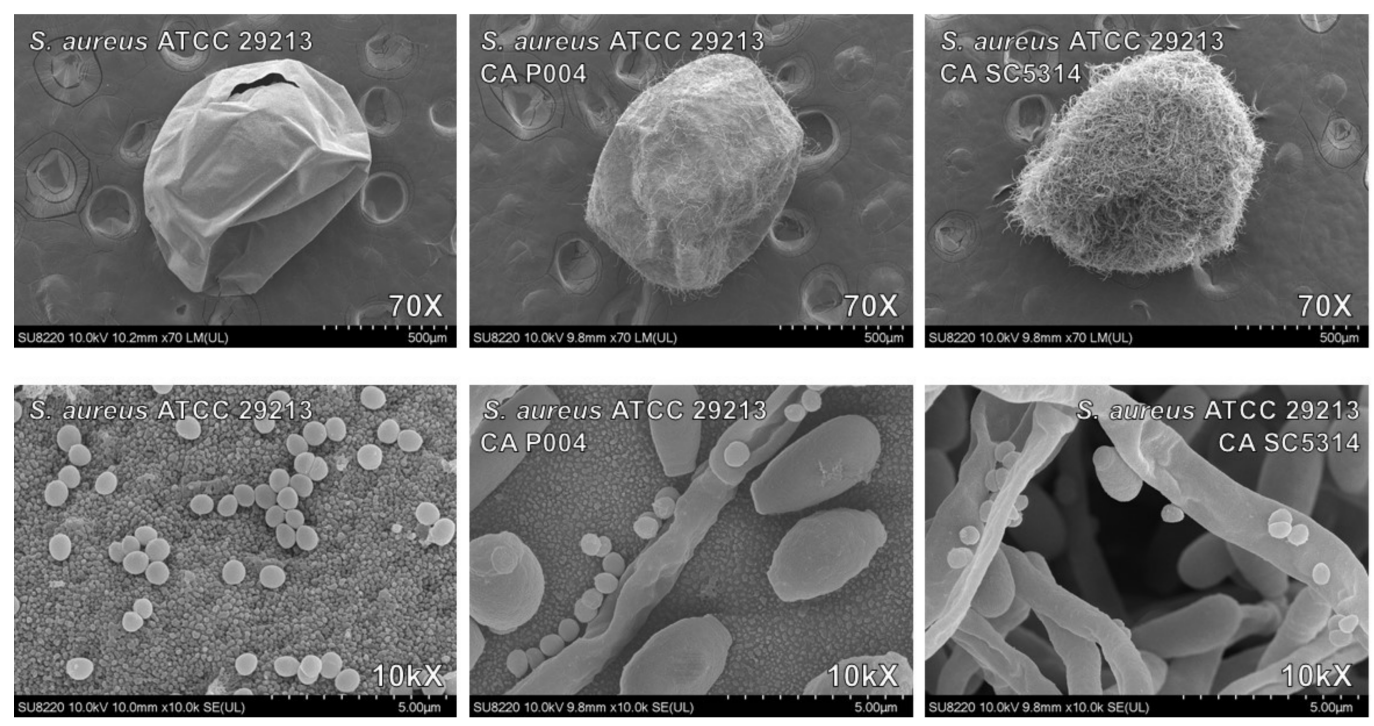

Figure 3. Scanning electron microscope graphs of biofilm morphology on fish embryo chorions. After 4-h adhesion and 24-h maturation, fish embryos that were infected with S. aureus ATCC 29213 only or that were co-infected with a low biofilm clinical strain P004 or wild-type SC5314 strains were used for SEM.

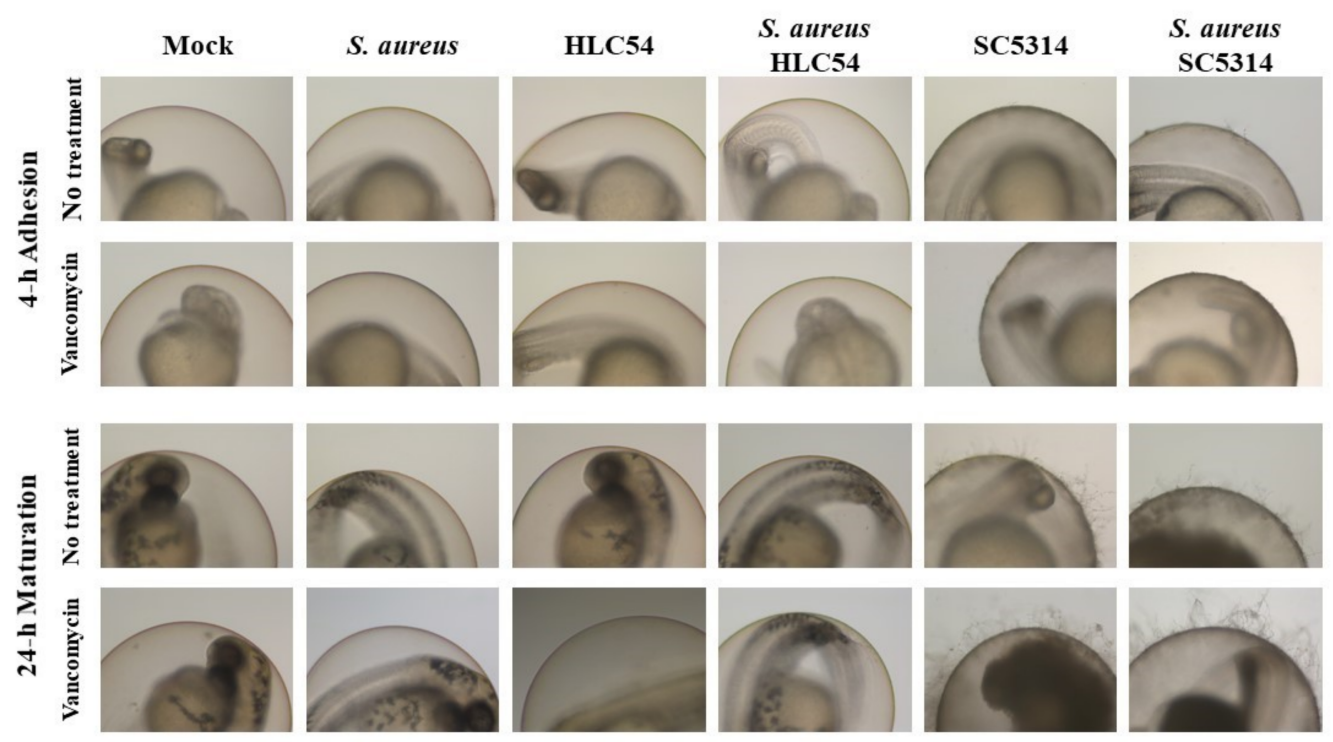

Figure 4. The morphology of mixed biofilm that are treated with S. aureus and C. albicans under vancomycin. After 4-h adhesion and 24-h maturation, fish embryos that were infected with S. aureus ATCC 29213, a hyphae-defective mutant HLC54, or wild-type SC5314 strains individually or mixed infected were photographed using a stereotactic microscope. Rows 2 and 4 share the same preparation of infected embryos but involve supplementation of $80 \mu \mathrm{g} / \mathrm{mL}$ vancomycin.

\subsection{Changes in S. aureus Toxin Genes in A Co-Biofilm}

S. aureus toxin genes were augmented by $C$. albicans in murine infection models, including $h l a$ (alpha hemolysin) and $h l g B$ (gamma hemolysin) [25]. If S. aureus formed cobiofilms with $C$. albicans on a filter membrane after 24-h maturation, there was a significant 
increase in expression of $h l a(7.06 \pm 2.19$ fold increase) and $h \lg B(8.12 \pm 2.64$ fold increase) in SC5314 group but not in hyphae-defective HLC54 group (hla: $1.54 \pm 0.30$ and $h \operatorname{lgB}$ : $2.09 \pm 0.81$ ) (Figure 5).

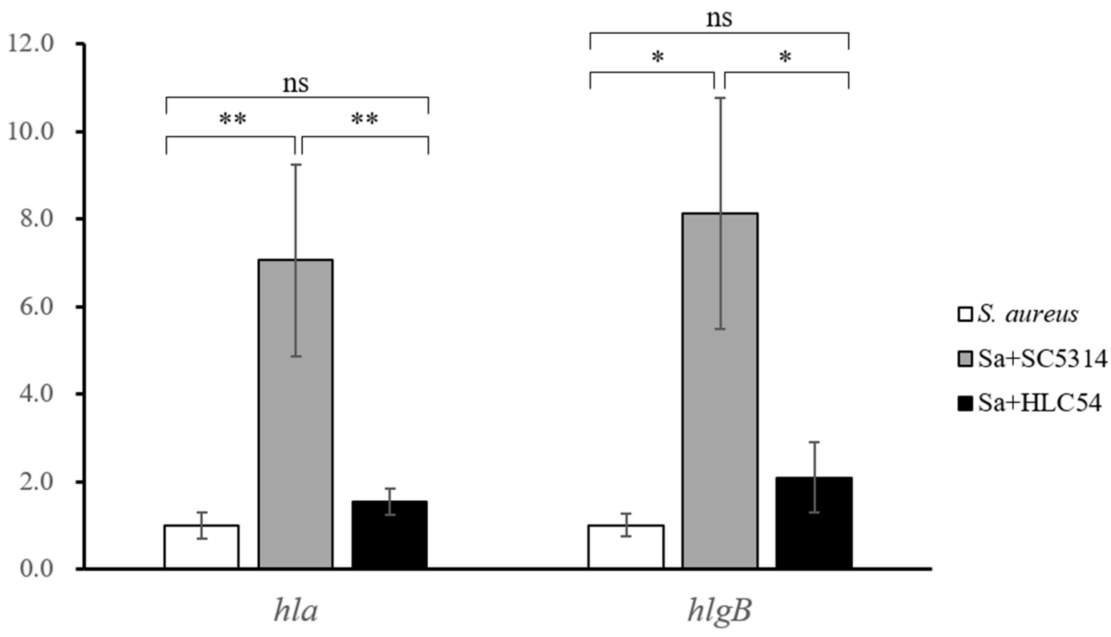

Figure 5. The upregulation of $S$. aureus toxin genes $h l a$ and $h l g B$ in a mixed biofilm that is formed by S. aureus and C. albicans SC5314. After 24-h maturation of biofilms on filter membranes, the in vitro biofilms that were formed with S. aureus only (SA, white bars), S. aureus co-cultivated with C. albicans SC5314 (SA + SC5314, gray bars), or with hyphae-defective mutant HLC54 (SA + HLC54, black bars) were washed twice, and total RNA was isolated and reverse transcribed to determine gene expression. Note: ${ }^{*} p<0.05,{ }^{* *} p<0.01$, ns $=$ not significant.

\section{Discussion}

This study demonstrates that the clinical outcome strongly supports the results for an in vitro enhanced virulence for candidemia by $S$. aureus. Within the monomicrobial C. albicans BSI group, more patients were found with parenteral nutrition and immunosuppressants, so this population was more immunocompromised. However, in comparison with this group, 22 mixed CA/SA BSI patients, enrolled from 264 mixed CA/bacterial BSIs (2003-2015), were associated with a higher SOFA score, increased 14-day and 30-day mortalities, and shorter median survival days. This demonstrates that $S$. aureus increases the virulence of $C$. albicans in BSI patients.

Zhong et al. reported that patients with mixed Candida albicans/bacterial bloodstream infections in China undergo mechanical ventilation for longer and stay longer in the ICU than patients with monomicrobial C. albicans BSIs, but there is no difference in 28-/60-day mortality [5]. The difference in mortality for mixed BSIs in different studies may be due to different bacterial pathogens. Staphylococcus is associated with higher mortality for animal hosts [6-11], but Enterococcus spp. negatively affects each other's virulence [26-28].

In terms of comorbidity, there is a higher incidence of renal insufficiency in mixed BSI group (Table 1). Patients who did not survive for 14 days had a higher prevalence of liver dysfunction and renal insufficiency and had almost four-times higher incidence of mixed CA/SA BSIs (Table 2). The authors demonstrated previously that a higher prevalence of renal insufficiency was associated with high-biofilm C. albicans isolates [17]. Herein, in vivo zebrafish embryo infection assay exhibited embryos co-infected with $S$. aureus and high-biofilm C. albicans survived less. Therefore, renal insufficiency is closely associated with mixed CA/SA BSI. In a 12-year period autopsies review, the most frequently involved deep parenchymal organ in candidiasis was the kidney [29]. Shin et al. reported that severe renal failure plays a major role in lethality for mice infected with C. albicans [30]. In an intravenous mouse infection model, kidneys were demonstrated to be the main target of C. albicans, though the upregulation of hyphae-associated genes was measured in the liver without visible hyphal invasion [31]. 
Our study results show that $S$. aureus significantly increases biofilm formation of C. albicans in the in vitro abiotic assay and in vivo surrogate zebrafish model. The increased biofilm of $C$. albicans and enhanced virulence of $S$. aureus are the reason for higher mortality in mixed BSIs. For the biofilm model with zebrafish eggs, only wild-type C. albicans provides $S$. aureus with protection against vancomycin rather than hyphae-defective mutant (HLC54). SEM images show that there is tight contact between the hyphae of C. albicans and $S$. aureus in a thick and multilayer biofilm, in which there is a matrix that is composed of an entangled polymeric substance during maturation that shields against antimicrobials.

This study shows that the SOFA score was significant higher within mixed CA/SA BSI patients, so interaction between these two pathogens probably exacerbates tissue damage and erosion during hyphal invasion. Our embryo infection result suggested C. albicans cobiofilm protects $S$. aureus against antimicrobials, and the major toxin genes of $S$. aureus are significantly upregulated, including $h l a$ and $h \lg B$, resulting in high mortality for $C$. albicans SC5314-infected embryos. An upregulation of virulence factors in S. aureus, including hla, $h \lg B$, enterotoxin family protein, staphylocoagulase, staphylococcal protein A, nucleases, intercellular adhesion proteins, fibronectin-binding proteins, drug-resistant genes glmU, murC, and $\operatorname{murD}$, and penicillin-binding proteins, was demonstrated by $\mathrm{Hu}$ et al. using cutaneous abscess and peritonitis murine models [25]. The increase in toxin production, especially exterotoxins, damages tissues and induces severe inflammation, which can cause multi-organ failure.

The study has some limitations. First, we acknowledge that insufficient information about follow-up blood cultures to confirm pathogen clearance was obtained in CA/SA BSI patients in this single-center retrospective study. However, these were critically ill patients with high mortality, and 12 of them $(12 / 22=54.5 \%)$ died within seven days after mixed CA/SA BSIs occurred. Second, the zebrafish has recently become an extremely powerful model organism in the context of Candida infections and host-pathogen interactions [32]. However, the drawback of the model is that the embryos were collected and grown at $30{ }^{\circ} \mathrm{C}$, which does not allow accurate mimicking of human infection.

\section{Conclusions}

Mixed CA/SA BSIs are associated with high morbidity and mortality in our hospital. The zebrafish embryo in vitro infection assay exhibits antibiotic tolerance in $S$. aureus if it attaches to the hyphae of C. albicans. Using in vitro assays, the production of $S$. aureus toxins in Candida co-biofilm possibly involves pathogenesis.

Supplementary Materials: The following are available online at https:/ / www.mdpi.com/article/10 .3390/jof7121099/s1, Table S1: The primer sets and TaqMan probes for the gene expression assay; Figure S1: The enhancement of $S$. aureus biofilm using culture supernatants of $C$. albicans.

Author Contributions: Y.-M.W. and P.-Y.H. contributed equally to the design and draft of the manuscript; Y.-M.W. contributed clinical data and performed analysis; Y.-C.C. and C.-H.L. performed the zebrafish experiments and gene expression assays; C.-H.L. and M.-C.H. conducted the in vitro biofilm experiments; Y.-C.C. assisted in editing the manuscript; J.-J.L. coordinated and supervised data collection and critically reviewed the manuscript; S.-H.W. contributed to the design and the analysis of data and wrote and edited the manuscript. All authors have read and agreed to the published version of the manuscript.

Funding: The study was supported by a grant from CGMHL to P.-Y.H. (CMRPG3K2401) and by grants MOST 109-2314-B-415-003 and 110-2320-B-182A-006-MY3 from the Ministry of Science and Technology of Taiwan to S.-H.W. and J.-J.L.

Institutional Review Board Statement: The study was approved by the institutional review board of the CGMHL (approval number: 201701182B0 and 202101345B0).

Informed Consent Statement: Not applicable.

Acknowledgments: The authors are grateful for the technical assistance of the Microscopy Core Laboratory, CGMHL. 
Conflicts of Interest: The authors declare no conflict of interest.

\section{References}

1. Pfaller, M.A.; Carvalhaes, C.G.; Smith, C.J.; Diekema, D.J.; Castanheira, M. Bacterial and fungal pathogens isolated from patients with bloodstream infection: Frequency of occurrence and antimicrobial susceptibility patterns from the SENTRY Antimicrobial Surveillance Program (2012-2017). Diagn. Microbiol. Infect. Dis. 2020, 97, 115016. [CrossRef] [PubMed]

2. Martinez Perez-Crespo, P.M.; Lopez-Cortes, L.E.; Retamar-Gentil, P.; Garcia, J.F.L.; Vinuesa Garcia, D.; Leon, E.; Calvo, J.M.S.; Galan-Sanchez, F.; Natera Kindelan, C.; Del Arco Jimenez, A.; et al. Epidemiologic changes in bloodstream infections in Andalucia (Spain) during the last decade. Clin. Microbiol. Infect. 2021, 27, e283-e289. [CrossRef]

3. Chen, X.C.; Xu, J.; Wu, D.P. Clinical characteristics and implications of mixed candida/bacterial bloodstream infections in patients with hematological diseases. Eur. J. Clin. Microbiol. Infect. Dis. 2020, 39, 1445-1452. [CrossRef] [PubMed]

4. Bouza, E.; Burillo, A.; Munoz, P.; Guinea, J.; Marin, M.; Rodriguez-Creixems, M. Mixed bloodstream infections involving bacteria and Candida spp. J. Antimicrob. Chemother. 2013, 68, 1881-1888. [CrossRef]

5. Zhong, L.; Zhang, S.; Tang, K.; Zhou, F.; Zheng, C.; Zhang, K.; Cai, J.; Zhou, H.; Wang, Y.; Tian, B.; et al. Clinical characteristics, risk factors and outcomes of mixed Candida albicans/bacterial bloodstream infections. BMC Infect. Dis. 2020, 20, 810. [CrossRef]

6. Peters, B.M.; Noverr, M.C. Candida albicans-Staphylococcus aureus polymicrobial peritonitis modulates host innate immunity. Infect. Immun. 2013, 81, 2178-2189. [CrossRef] [PubMed]

7. Todd, O.A.; Fidel, P.L., Jr.; Harro, J.M.; Hilliard, J.J.; Tkaczyk, C.; Sellman, B.R.; Noverr, M.C.; Peters, B.M. Candida albicans augments Staphylococcus aureus virulence by engaging the staphylococcal agr quorum sensing system. mBio 2019, 10, 19. [CrossRef]

8. Schlecht, L.M.; Peters, B.M.; Krom, B.P.; Freiberg, J.A.; Hansch, G.M.; Filler, S.G.; Jabra-Rizk, M.A.; Shirtliff, M.E. Systemic Staphylococcus aureus infection mediated by Candida albicans hyphal invasion of mucosal tissue. Microbiology 2015, 161, 168-181. [CrossRef]

9. Vila, T.; Kong, E.F.; Montelongo-Jauregui, D.; Van Dijck, P.; Shetty, A.C.; McCracken, C.; Bruno, V.M.; Jabra-Rizk, M.A. Therapeutic implications of C. albicans-S. aureus mixed biofilm in a murine subcutaneous catheter model of polymicrobial infection. Virulence 2021, 12, 835-851. [CrossRef]

10. Holt, J.E.; Houston, A.; Adams, C.; Edwards, S.; Kjellerup, B.V. Role of extracellular polymeric substances in polymicrobial biofilm infections of Staphylococcus epidermidis and Candida albicans modelled in the nematode Caenorhabditis elegans. Pathog. Dis. 2017, 75, 15. [CrossRef]

11. Sheehan, G.; Tully, L.; Kavanagh, K.A. Candida albicans increases the pathogenicity of Staphylococcus aureus during polymicrobial infection of Galleria mellonella larvae. Microbiology 2020, 166, 375-385. [CrossRef]

12. Esher, S.K.; Fidel, P.L., Jr.; Noverr, M.C. Candida/Staphylococcal polymicrobial intra-abdominal infection: Pathogenesis and perspectives for a novel form of trained innate immunity. J. Fungi 2019, 5, 37. [CrossRef]

13. Scherlach, K.; Hertweck, C. Chemical mediators at the bacterial-fungal interface. Annu. Rev. Microbiol. 2020, 74, 267-290. [CrossRef]

14. Nogueira, F.; Sharghi, S.; Kuchler, K.; Lion, T. Pathogenetic impact of bacterial-fungal interactions. Microorganisms 2019, 7, 459. [CrossRef]

15. Wang, S.H.; Chen, C.C.; Lee, C.H.; Chen, X.A.; Chang, T.Y.; Cheng, Y.C.; Young, J.J.; Lu, J.J. Fungicidal and anti-biofilm activities of trimethylchitosan-stabilized silver nanoparticles against Candida species in zebrafish embryos. Int. J. Biol. Macromol. 2020, 143, 724-731. [CrossRef]

16. Deng, F.S.; Lin, C.H. Identification and characterization of ORF19.1725, a novel gene contributing to the white cell pheromone response and virulence-associated functions in Candida albicans. Virulence 2018, 9, 866-878. [CrossRef]

17. Lu, J.J.; Lo, H.J.; Wu, Y.M.; Chang, J.Y.; Chen, Y.Z.; Wang, S.H. DST659 genotype of Candida albicans showing positive association between biofilm formation and dominance in Taiwan. Med. Mycol. 2018, 56, 972-978. [CrossRef] [PubMed]

18. Chen, Y.Z.; Yang, Y.L.; Chu, W.L.; You, M.S.; Lo, H.J. Zebrafish egg infection model for studying Candida albicans adhesion factors. PLoS ONE 2015, 10, e0143048. [CrossRef]

19. Jones, A.E.; Trzeciak, S.; Kline, J.A. The Sequential Organ Failure Assessment score for predicting outcome in patients with severe sepsis and evidence of hypoperfusion at the time of emergency department presentation. Crit. Care Med. 2009, 37, 1649-1654. [CrossRef] [PubMed]

20. Wu, Y.M.; Huang, P.Y.; Lu, J.J.; Shie, S.S.; Ye, J.J.; Wu, T.S.; Huang, C.T. Risk factors and outcomes of candidemia caused by Candida parapsilosis complex in a medical center in northern Taiwan. Diagn. Microbiol. Infect. Dis. 2018, 90, 44-49. [CrossRef] [PubMed]

21. Lo, H.J.; Köhler, J.R.; DiDomenico, B.; Loebenberg, D.; Cacciapuoti, A.; Fink, G.R. Nonfilamentous C. albicans mutants are avirulent. Cell 1997, 90, 939-949. [CrossRef]

22. Kao, C.Y.; Wu, H.H.; Chang, S.C.; Lin, L.C.; Liu, T.P.; Lu, J.J. Accurate detection of oxacillin-resistant Staphylococcus lugdunensis by use of agar dilution. J. Microbiol. Immunol. Infect. 2021, 2, 9. [CrossRef] [PubMed]

23. Reed, B.; Jennings, M. Guidance on the Housing and Care of Zebrafish Danio Rerio; Royal Society for the Prevention of Cruelty to Animals: Southwater, UK, 2011.

24. Kong, E.F.; Tsui, C.; Kucharikova, S.; Andes, D.; Van Dijck, P.; Jabra-Rizk, M.A. Commensal protection of Staphylococcus aureus against antimicrobials by Candida albicans biofilm Matrix. mBio 2016, 7, 16. [CrossRef] [PubMed] 
25. Hu, Y.; Niu, Y.; Ye, X.; Zhu, C.; Tong, T.; Zhou, Y.; Zhou, X.; Cheng, L.; Ren, B. Staphylococcus aureus synergized with Candida albicans to increase the pathogenesis and drug resistance in cutaneous abscess and peritonitis murine models. Pathogens 2021, 10, 1036. [CrossRef]

26. Cruz, M.R.; Graham, C.E.; Gagliano, B.C.; Lorenz, M.C.; Garsin, D.A. Enterococcus faecalis inhibits hyphal morphogenesis and virulence of Candida albicans. Infect. Immun. 2013, 81, 189-200. [CrossRef]

27. Krishnamoorthy, A.L.; Lemus, A.A.; Solomon, A.P.; Valm, A.M.; Neelakantan, P. Interactions between Candida albicans and Enterococcus faecalis in an organotypic oral epithelial model. Microorganisms 2020, 8, 1771. [CrossRef]

28. Graham, C.E.; Cruz, M.R.; Garsin, D.A.; Lorenz, M.C. Enterococcus faecalis bacteriocin EntV inhibits hyphal morphogenesis, biofilm formation, and virulence of Candida albicans. Proc. Natl. Acad. Sci. USA 2017, 114, 4507-4512. [CrossRef] [PubMed]

29. Parker, J.C.; McCloskey, J.J.; Knauer, K.A. Pathobiologic features of human candidiasis. A common deep mycosis of the brain, heart and kidney in the altered host. Am. J. Clin. Pathol. 1976, 65, 991-1000. [CrossRef]

30. Shin, J.C.; Jeon, Y.J.; Park, S.M.; Seo, K.S.; Shim, J.H.; Chae, J.I. Mechanism underlying renal failure caused by pathogenic Candida albicans infection. Biomed. Rep. 2015, 3, 179-182.

31. Hebecker, B.; Vlaic, S.; Conrad, T.; Bauer, M.; Brunke, S.; Kapitan, M.; Linde, J.; Hube, B.; Jacobsen, I.D. Dual-species transcriptional profiling during systemic candidiasis reveals organ-specific host-pathogen interactions. Sci. Rep. 2016, 6, 36055. [CrossRef] [PubMed]

32. Rosowski, E.E.; Knox, B.P.; Archambault, L.S.; Huttenlocher, A.; Keller, N.P.; Wheeler, R.T.; Davis, J.M. The zebrafish as a model host for invasive fungal infections. J. Fungi 2018, 4, 136. [CrossRef] [PubMed] 